\title{
LA CARICA DELLE SONDE RADIOAT'TIVE IN PRESENZA DI VENTO
}

\author{
R. Cialoea - A. Lo Struo t - G. Zavotelli
}

Nelle condizioni naturali il funzionamento delle sonde radioatlive, destinate alla misura del polenziale elellrico, i notorianente perlurbalo dalla presenza dei movinenti dellaria: guesta considerazione ei indusse a suo tempo a studiare l'effelto della presenza della carica spaziale sul valore del potenziale di equililuro, sia quando l'aria cireostante alla somda $i$ in quiele ('), sia quando la stessal $b$

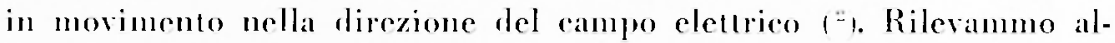
lora in qual morlo il potenziale assunto dalla sonda fosse influenzato dalla velocili della corrente d'aria, e le conclusioni dedotle valgono per le misure di polenziale effetluahili con le sonde, finché il campo eletrieo si mantiene rostante nel tempo.

Ma allorehe il rampo elettrico 2 variabile, im importante lo studio

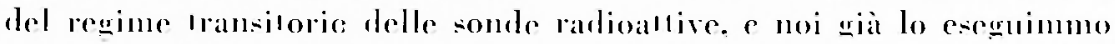
per le combizioni di aria calma f"I. Indubliamente i movimenti dell'aria si ripereuotono sulle morlaliti di carica e di scariea, e l'attitudine della sonda a seguire le rariazioni del campo is presumilibmente influenzala dalla velocila della corrente d'aria rehe la investe ${ }^{\circ} \%$ Da (ii) ennere lopportunili di integrare il ciclo delle nostre ricerche sul funzionamento delle sonde radioatlive nel condensatore piano con lo studio dellandanento dei fenomeni transitori in presenza di vento.

1 questo scopo alhliamo elahorato aleune consilerazioni teoriche. riferendoci al consueto modello di sonda, gia adottato nelle precedenti ricerche, rappresentala da uno strato ionizzante esteso, piano c sotlile, parallelo alle armalure di un condensalore indefinito. ael quale condensatore flusce una corrente d'aria uniforme e parallela alle linee di eampo. Ci siamo peraltro linniali a considerare i due casi estremi in eui la relocita dell'aria issai piecola o assai grande rispelto a quella assunta dagli ioni sotto la sola azione del rampo. wiaccho lale considerazione ci i sembrala sufliciente a lumegriare le linee renerali del lenomeno. 


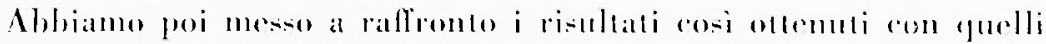
di una serie di esperienze rombleste sul romportamento. in regine transitorio. delle sombe reali solloposte all'azione del rento in wu condensalore piano.

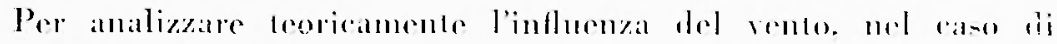

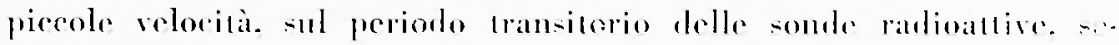

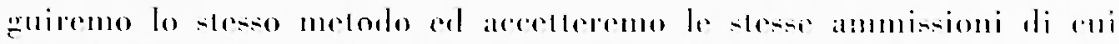



Fig. I al precentente lasoro relativo al regime lran-

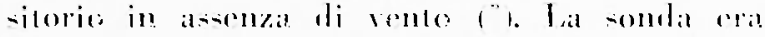
allora schemalizzala in lorma di strates entl.

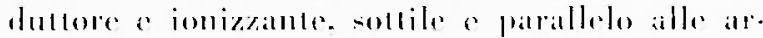

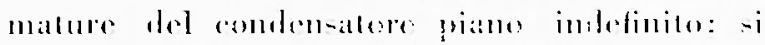

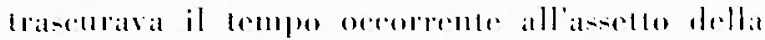

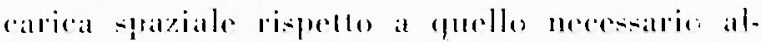

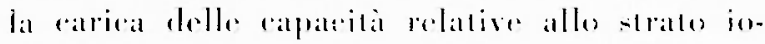

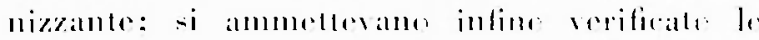

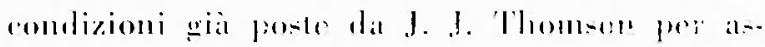

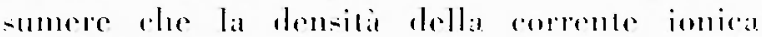
losie proporzionale al fuadrato dellat dillw.

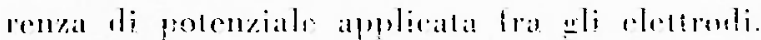

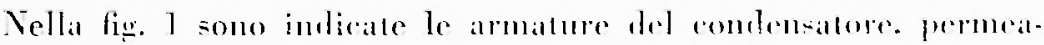
bili alla corrente d'aria nniforme di volocita g. o lo slrato ionizzante:

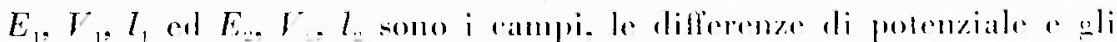

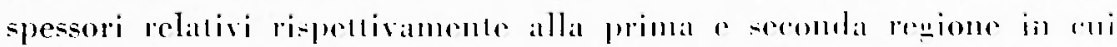
lo spazio intermo al comelensatore i dision dallo stato ionimante.

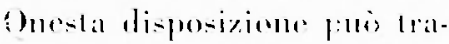

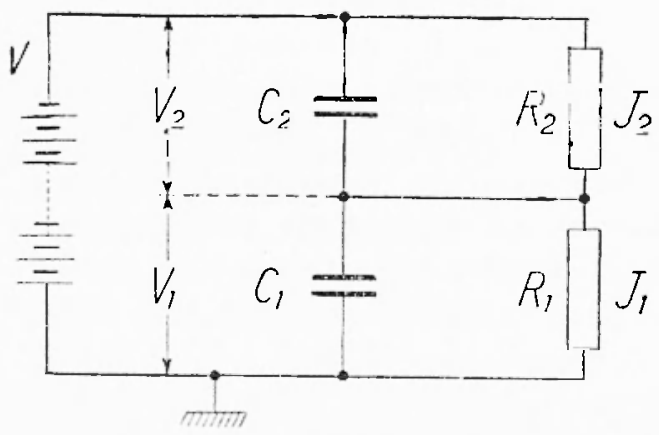

lis. 2

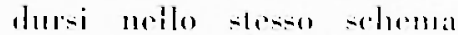
equivalente gia ulilizzaln nella precerlente ricereral rappresentato in fig. 2: C.. k. J c C.. R. . I rappre. sentamb rimpltivamente le capracila della somba c dello strmmento di misura per milit a serione verso l'una 0 l'alloal atrmallura, le lexisemze apparenti della primat ereombla regione e le 
densila di corrente ioniea allaberso la prima e secombla regione, densili rhe per le ipoles da noi pose si suppongono approsimali-

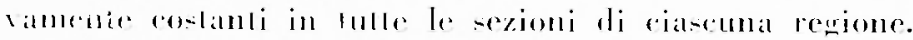

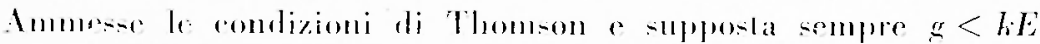

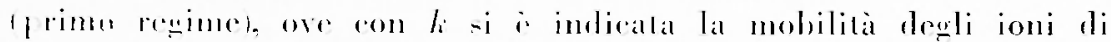

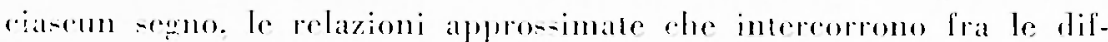
ferenze di polmziale e le densibi di corrente ionica ai rapi della frima revione. in rui si mosome solo ioni posilivi, e rispellisamente della secombla. in eni si muovone solo ioni newalivi, sono le seguenti.

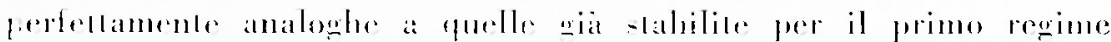
nel me-tre presedente lavoro

$$
\begin{aligned}
& \boldsymbol{r}=\frac{1}{h_{1}}\left[\frac{2}{3} \sqrt{3 . r_{1} l_{1} h_{1}}+g_{1}^{\prime}\right\rfloor, \\
& r_{z}=\frac{1}{l_{z}}\left|\begin{array}{l}
2 \\
3
\end{array}\right| 8+\sigma_{z}: l_{i}-g l_{2} \mid .
\end{aligned}
$$

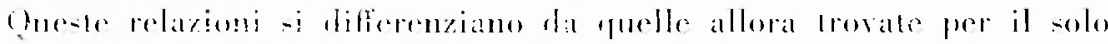

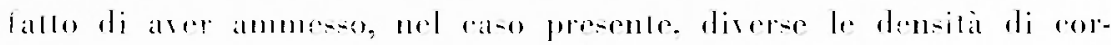

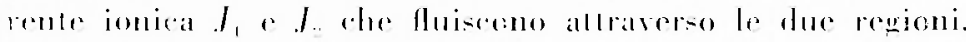

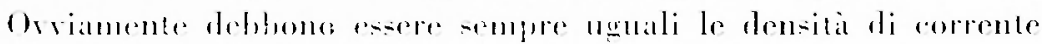

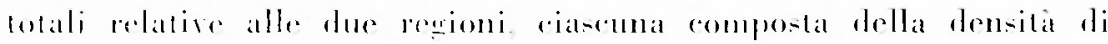

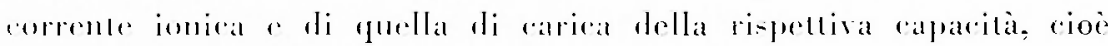

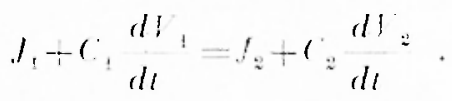

Rirordande the la ditlesenza di potenziale costante applieala al confentalere

$$
1 ;-\ldots 1 ;-\cdots
$$

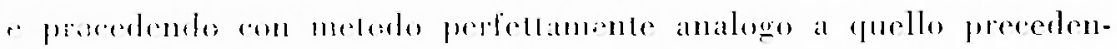

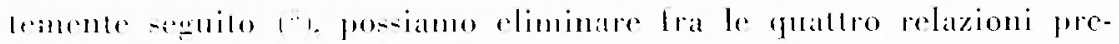


lilinenriale

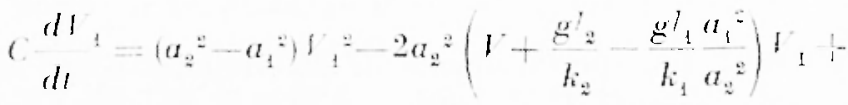

$$
\begin{aligned}
& +a_{2}=\left(1+21 \frac{g^{2} z}{l_{2}}+\frac{g^{2} a^{2}}{l_{2}^{2}}-\frac{g^{2} i_{1}^{2}}{a_{1}^{2}}\right)^{15}
\end{aligned}
$$

in rui -i i fere - omplicila posto

$$
C=C_{1}-C_{2} \text {. }
$$




$$
a_{1}=\sqrt{\frac{9}{32 \pi} \cdot \frac{b_{1}}{l_{1}:}}, \quad \quad a_{0}=\sqrt{\frac{9}{32 \pi} \frac{b_{2}:}{l_{2}} \cdot[7]}
$$

Guesta equazione. ele per $g-0$ si rilue owiamente a quella corrispontente in assenzal di vento ("), ammette un integrale generale di identical lormal. cioc

$$
\frac{l_{1}-\frac{\rho}{1}}{l_{1}-a}=.1 e^{-t T}
$$

ove $t$ c una rostante di integ̣razione determinabile in hates al va-

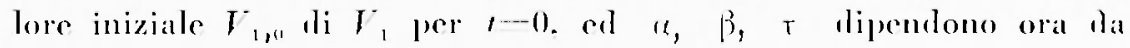
$g$ e asimmono le espressioni

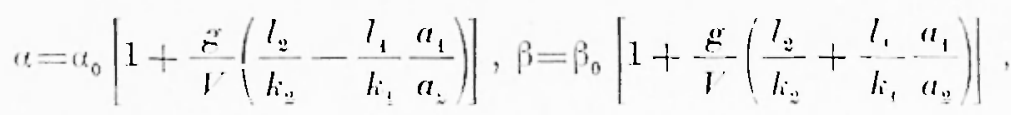

$$
\begin{aligned}
& \tau=1+\frac{g}{V}\left(\frac{l_{2}}{l_{z}}-\frac{l_{1}}{l_{i_{1}}}\right)
\end{aligned}
$$

Le erandezac $\theta_{0}$. $\sigma_{0}, T_{0}$ non sono altro che le corriopondenti wrandezze per le condizioni di allia calma (")

$$
\sigma_{0}=\frac{r}{1-\frac{a_{1}}{a_{2}}}, \beta_{0}=\frac{V}{1+\frac{a_{1}}{a_{2}}}, \tau_{0}=\frac{C}{2 a_{1} a_{2} V} .
$$

Poiche la soluzione coincide formalmente ron quella wia othemuta in assenza di vento. posiono senz'altro tratserirsi al raso presente lutte le considerazioni allora derlotte per l'amdamento del polenziale in funzione del tempo. purehe si lengal presente la dipendenza delle u, fi, $\tau$ dalla velocila del vento. In particolare il potenziale di equilibrio $r_{1, s}=\hat{\beta}$, che si oftiene dalla [8] facendo tembere 1 all’infinito, roincide, come era da frevedersi, ron quello determinato a sto temino (i).

A noi interessa piu specifieamente li determinare come le pice cole velocita della corrente d'aria influiscano sul tempo richiesto dalla sondal per la carica o lat scalrica. Assali fiù espressivo della costante

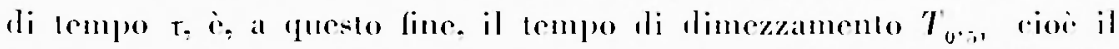
tempo occorente perehe la sonda superi meta dellintervallo la il potenziale di regime $V_{1, s}$ e quello iniziale $V_{1,0}$, che come lu a sto 
tempo mostrato Th, $i$ Melerminabile a partire dalla soluzione $[8]$ nella forma

$$
T_{0.5}=\mathrm{r} \ln \left[1+\frac{V_{1,5}-\alpha}{V_{1,0}-\alpha}\right] .
$$

Per lo sopo anzideto is sufficionle considerare la derivata di

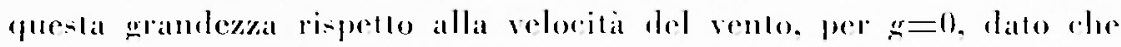
la relocita slessa is sempre presa ats-ai piecola. Il raleolo materiale, lenule presenti le [9], [10], non offere diffeolla: ad es per la rariea

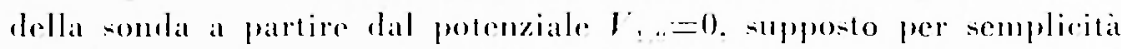
$h_{1}=-h_{i}$, la variazione pereentuale elementare del tempo di dimeazamento in rappoto alla variazione della velocita del rento, finche que sta i assai piecolat as-mome la forma

$$
\begin{aligned}
& \left|\frac{1}{T_{0.5}} \frac{d T_{0.5}}{d g}\right|_{z=0}=
\end{aligned}
$$

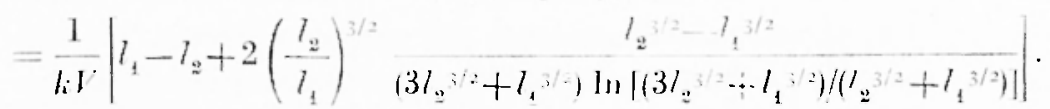

Per $l=l$ questa espresione si amullate ciod esiste ma posizione intermedia lra le amature in cui il tempo di dimex. zamenlo risulta indipendente dalla velocita del vento: questa cile-

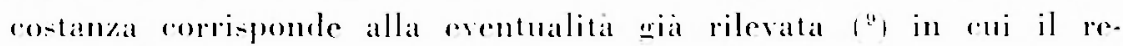
rime di carica della sonda e fucllo stesos di carica di un condene satore attraterso ad mat resi-tenzal puramente ohmica. Al parsagerio attraverso tale posizione intermedia l'espressione in parentesi a secondo membro cambia senno. Astumento per te come valore approsimalo medio lra le due specie di ioni son we.s. premlendo per lo spesore del condensalore $l_{1}+l,-60$ an e per la differenza di potenziale applieata $1=1.5$ ure.s. la $[137$ fornise per le tre posizioni della sonda $l_{1}-15: 30: 15 \mathrm{~cm}$. rispetlisamente i valori + $1.3 .10^{-}:$: 0: 2.1.10-3. Ve risulta che, allorehe la sonda e prossima alla prima oppure alla seconda armalura, lammento della velocita del vento porla al un aumento o an ma diminuzione del fempen di dimeramento. per quanlo questa ultima sia in rombonlo assali piò lieve: via via rhe la somda si porta verso il contro del condensalore l'infuenza del vento si attenua sempre più sino a rendersi insen. siliile.

F' chiaro poi che se la velocita del vento in invere di verso ron. trario a quelio supposto in lige. I. rios is romeorle con il campo elet- 


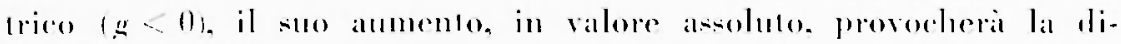
minurome del tempo di dimezamento in prosimita della prima armatura e l'aumento in proseimita della secomblas in altere parole. fissala ma certa posizione della sonda, la variazione del tempo di dimeezamento si inverte con l'invertirsi del verso della velociti del vento.

Youliamo ora comsilerare il comportamento della somla durambe

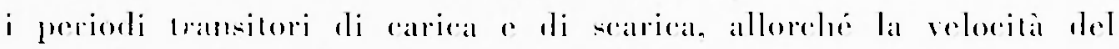

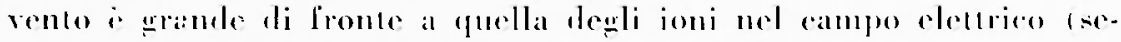

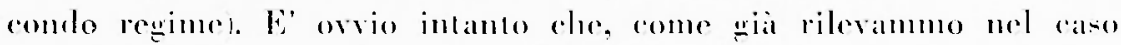

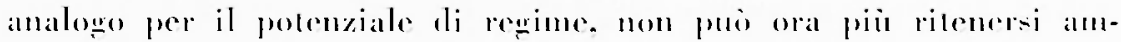
missibile la combizione di Thomson, perele oli ioni rengomo asporlati dallo strato ionizzanle, per aziome del movimento dell'aria. ma-



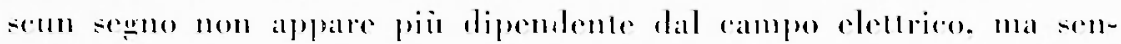

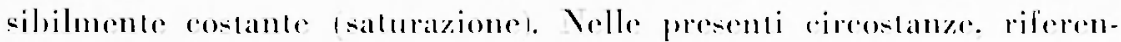
doei come al solito alle posizioni della liğ. l. la prima repione risulta completamente volat di carichere non esomelo per ipotesi il campose elettrieo al limite dello strato ionizzante sufficiente a permellero che gli ioni positivi risalgamo la corrente dellaria: wli slessi ioni positivi rengono traportati nella secomba regione insiene con quelli newalivi. e si muovomo ron questi ultimi verso la secomba ar-

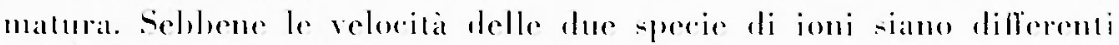
c late precisamente da $g$ he rispellivamente per grli ioni newaltisi e positivi. le due densita di corrente ioniea $J$ c $J$ risultano sem-


il regime di salurazione: precisamente le slesere sono dale dal prodotto dell'intensiti di ionizzarione nello strato per lo spesome sia pure supposto infinitesimo, di questultimo. Mancamdo nol seondo regime ogni corrente di combluzione ionica allawerso la prima regione, ogni variazione del potenziale dello strato intermedio deve pre supporre tura corrente di carical o seariea allraverso la secomba regione, oviamente determinata dalla dillerenza tra le correnti io-

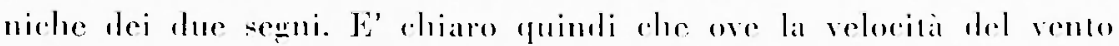
sia presa sufficiente al assicurare la salturazione, risultamberempre tumali in valore assoluto tali due correnti, non potra sussistere aleun processo di carica o scarica dello strato. La costamte di lempo del fenomeno deve quindi necesariamente tembere all'infinito, allorelue 


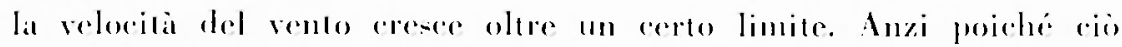
deve verifiealrsi sia quamde la eorecente d'aria é coneorde sia quando

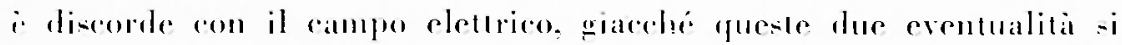
diflerenziano solo per essere le cariehe dei due segni o tulle nella frima " tutte rispettivamente nella secombla regione, is presumibile che lat cosiante di tempo cresca con la velociti del rento, indipendentemenle dal suo sonno.

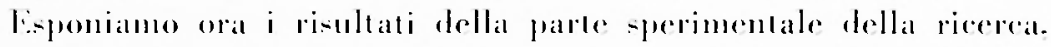
rhe iu diretla a determinare il tempe di dimezamente proprio di una sonda reale. Siluala in diverse posizioni nell'interno del condensatore. e in presenza di una corrente d'aria fluente parallelanente alle line di forza. Furono adotlati il dispositivo ed il metodo di

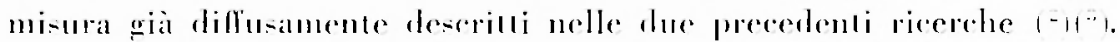

Il campo eleterien era penerate dalle due prambiamature di

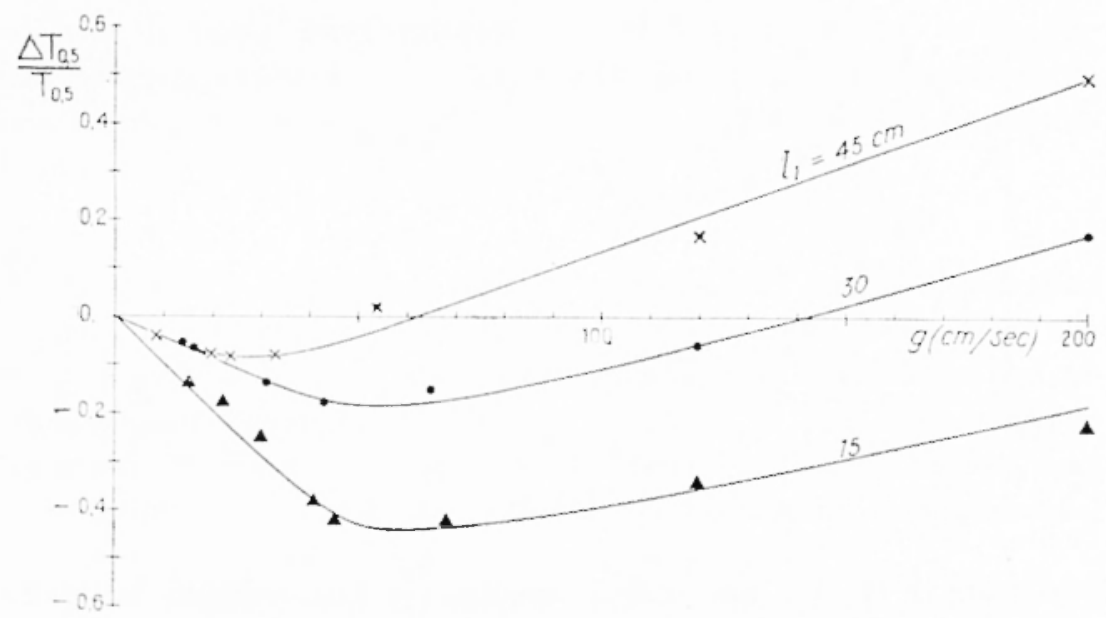

Fig. 3

rete melalliea poste alla distamza di 60 com: una corrente d'aria proLolta dalla piocola walleria acoodinamica fluiva lungo le linee del -ampo. Le esperienze furono contotte per vari valori della velocila

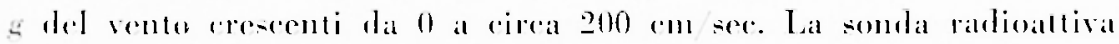
impregalta era il solito enbetto di otlone di $15 \mathrm{~mm}$ di spigolo. che portat -11 una delle fiace un disehelto metallieo ricoperto eleltroliticamente di polonio. La sonda reniva collocata nell'interno del 
contensalore in tre diverse posizioni, cioe 15,30 e 45 em dall'armalura a potenziale zero.

Alemi risultati delle determinazioni sperimentali del tempo di dimezamento nel caso della cariea della sonda a partire dal polenziale $V_{1, n}=0$ sono rappresentati nel diagaramma di fig. 3 , relativo alle tre posizioni della sonda. In orlinate sono riporlati wli incrementi, dovuti allazione del vento. del tempo di dimezamento in rapporlo allo stesso tempo in assenza di rento, cioi $\Delta T_{0,5} / T_{0,5}=\mid\left(T_{0,5}\right)_{n}$ $\left(T_{0,)_{0}}\right) /\left(T_{0,5}\right)_{0} ; \quad$ in ascisse le velocitia del vento. In queste esperienze il vento fluiva in senso roncome al campo verso la prima armattura, inixialnente collegala alla somela, cioe in rerso contrario a quel-



Fig. 1

lo indicato in fige. 1. Come si vede. mente per forti velocita del verbo il tempo di dimezzamento arese con il reserere della velociti del vento. per piecole velocili invece diminuisee, in maniera più pronunciala allorehe la sonda si trowa in prosimila della prima armale tura. e sempere meno spiecalamente invece via via che si viene a trovare in punti piì lontani.

Nel liagramma di fig. 4 sono rifortati invere pli stesisi incrementi perentuali del lempo di amezamento per la posizione $l_{1}=15 \mathrm{~cm}$. in cui la dipentenza della variazione della velocita del vento e piu spiceata, in confronto a fuelli relativi alla stessa posizione, ottenuli però invertento la vébeitì del vento, in modo che questo, discorale 
ora al campo elettrico, fluiva ver-o la secomela armatura. Mentre per forti velocita del rento, indipendentemente dal loro senso, il tempo di dimezzamenlo ammenta sempere, per piccole velocila invere l'inversione del loro senso inverte anche il segno della variazione del empo di dimezamento.

Il diagramma di figr. I ci illu-tra inoltre ma interessante paslicolarila del comportamento del lempo di dimezamento per valori intermedi delle velociti del fluson di aria, corripomente all'in-

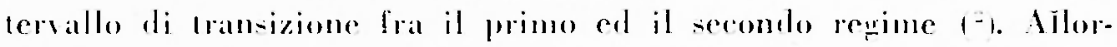
che il vento fluiser diseorlemente al aumpo eleltrico, all'aumentare della -na veloeita il lempe di dimezamento presenta prima un mat-simo e poi un minimo, oltre il fuale ripremele a crescere indefui-

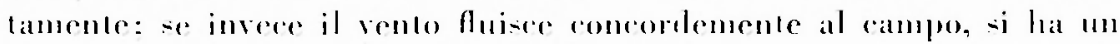
mieo minimo. In ogni raso quimeli risulta che il tempo di dimezatmento assume nell'intervallo di transizione fra il primo ed il se. comelo regime un valore minimo. Tuttavia fuesti risultati sperimentali non delobuno esere valutati oltre il loro signifiealo, anche perche, come acenneremo in seguito. sull'andamento del tempo di dimexamento agiseono in maniera rilevante la loma e le dimensioni lellal $-m$ mila.

Mettemlo a rallironte $\mathrm{i}$ risultati ora riportati con le romelusioni leoriche. si riseontra soddistacentemente verifieata la circostanza rhe

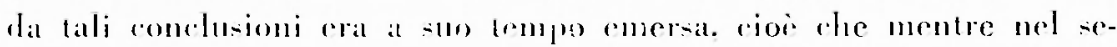

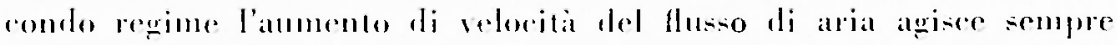

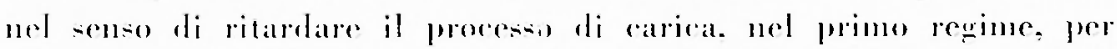
relociti prosime a zero. la presenza led rento pure a secomda del - wo veroo. alerelerare o ritamiare lo stesso processo.

Eaminando imvere il romportamento per pireole velocita del rento. del tempo di dimezanmento nelle varie posizioni della sonda. $\therefore$ risentra che mentre la previsione teoriea indiea ma inversione dell'andamento per velocita del rento eresenti a seromela che lat sonla s con-ibleri prosima allma od allallra armatma. a detta dellesperienza rio non si verifiea. Sorevera il fondalo dubbio che la rat

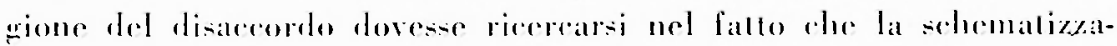
xione alolotata di ma sombla in forma di strato ionizzante imdefunilo non fosse, in queste rifrostanze. piu idonea a rappresentalue la sombla reale 1 (").

Per chiarire questo punto abhiamo condotle aleme allre expe- 
ricnze per le tre seswe posizioni della somdat per vento comeorde alla direzione del campo elettrieo. e limilatamente a hasse velocila del

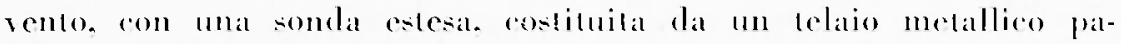
rallelo alle armature del condensatore, della sezione di $720 \mathrm{~cm}^{2}$, porlimle 27 sonde ( $^{\prime \prime \prime}$.

I valori della derivala dell'increnento perentuale del tempo di dimezzamento cosi oltenuli sono messi a rathronto nellat seguente labella eon i corrispondenti rilevabili per la sonda normale dal diagramma di fig̣. I. e ron quelli dedolli in via teorical.

\begin{tabular}{|c|c|c|c|}
\hline$l_{1}$ & 1.5 & 30 & $15, \mathrm{~m}$ \\
\hline somela mormale & $+8,0 \cdot 10^{-3}$ & $+3,5 \cdot 10^{-:}$ & $-3,5 \cdot 10: 3$ \\
\hline somda estesil & $+28 \cdot 10=$ & $+1,2 \cdot 10^{-:}$ & $-2,9.10 \cdots$ \\
\hline leoria & $1,3 \cdot 10^{-i}$ & 0 & $-2,1,10^{-3}$ \\
\hline
\end{tabular}

Come si vede la teoria $i$ in aceordo. che puo ritenersi almeno qualitativamente soddisfacente. con i risulati sperimentali relativi alla sonela estesa, rio che dinostra rome. per piecole velocita del rento. forma e dimensioni della sonda reale possano esplieare un efledto rilevante sulliandamento della rarical.

Riassumendo, le conclusioni di mangior rilievo ehe comerenono dal nostro studio ei sembramo le sesuenti:

$1)$ Per piecole velocita del vento i processi di rarical o di scalrica della somda risultano rallentati od aceelerati a seconda del reres in cui fluise la corrente d'aria. E' prevedibile pereio rhe la presenza di piccole flutuluzioni dell'aria intomo alla sondal posis in media non alterare, se non in misura assai ridolta, il tempo richiesto dalla sonda per ragegiungere il polenziale di equilibrio.

2) Per forti velociti del rento, invece, il tempo richiesto pere l'equilibrio risulta sempre aumentato qualumque sia la direzione del 
vento, rese in queste comblizoms il movimento dell'aria riduce sostanzialmonle l'efficienza dell'erenalizatore di polenziale.

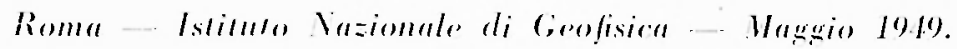

\section{RISSTU N\%}

Si sturlia leffente di mon corrente d'arien parallela alle limee del

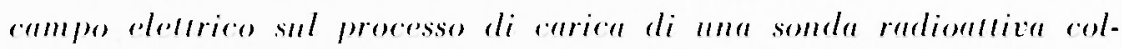

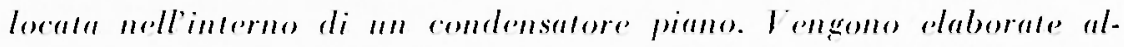
clune considerasioni aroriche per il modello della somda sollite e la-

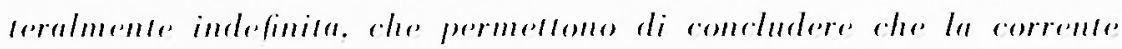

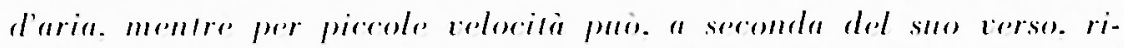

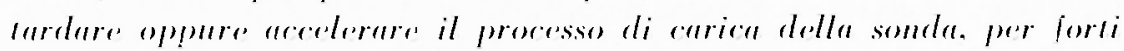

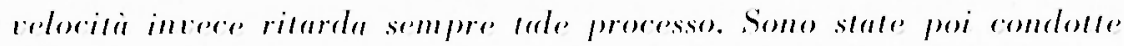
esperiense i rai risuleali concordano con le deduzioni teoriche. ed illustrano anche lefjelle delle dimensioni della somde reale.

\section{ISIIBI.IOT;R:IIIA}

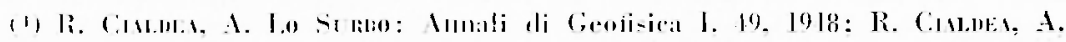

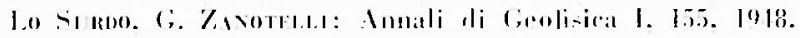

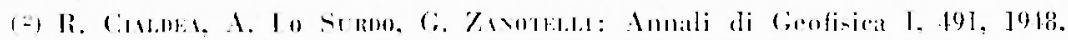

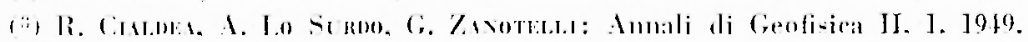

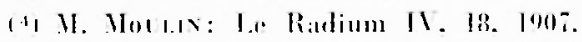

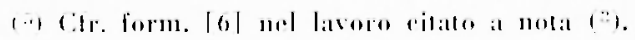

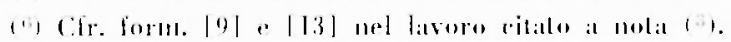

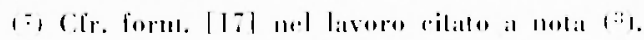

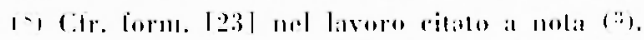

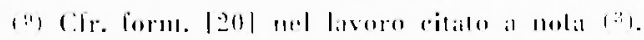

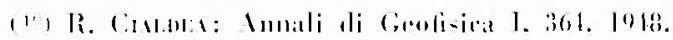

